\title{
STRUCTURE OF RINGS WITH CERTAIN CONDITIONS ON ZERO DIVISORS
}

\author{
HAZAR ABU-KHUZAM AND ADIL YAQUB
}

Received 4 May 2004; Revised 17 September 2004; Accepted 24 July 2006

Let $R$ be a ring such that every zero divisor $x$ is expressible as a sum of a nilpotent element and a potent element of $R: x=a+b$, where $a$ is nilpotent, $b$ is potent, and $a b=b a$. We call such a ring a $D^{*}$-ring. We give the structure of periodic $D^{*}$-ring, weakly periodic $D^{*}$-ring, Artinian $D^{*}$-ring, semiperfect $D^{*}$-ring, and other classes of $D^{*}$-ring.

Copyright (c) 2006 Hindawi Publishing Corporation. All rights reserved.

\section{Introduction}

Throughout this paper, $R$ is an associative ring; and $N, C, C(R)$, and $J$ denote, respectively, the set of nilpotent elements, the center, the commutator ideal, and the Jacobson radical. An element $x$ of $R$ is called potent if $x^{n}=x$ for some positive integer $n=n(x)>1$. A ring $R$ is called periodic if for every $x$ in $R, x^{m}=x^{n}$ for some distinct positive integers $m=m(x)$, $n=n(x)$. A ring $R$ is called weakly periodic if every element of $R$ is expressible as a sum of a nilpotent element and a potent element of $R: R=N+P$, where $P$ is the set of potent elements of $R$. A ring $R$ such that every zero divisor is nilpotent is called a $D$-ring. The structure of certain classes of $D$-rings was studied in [1]. Following [7], $R$ is called normal if all of its idempotents are in $C$. A ring $R$ is called a $D^{*}$-ring, if every zero divisor $x$ in $R$ can be written as $x=a+b$, where $a \in N, b \in P$, and $a b=b a$. Clearly every $D$-ring is a $D^{*}$-ring. In particular every nil ring is a $D^{*}$-ring, and every domain is a $D^{*}$-ring. A Boolean ring is a $D^{*}$-ring but not a $D$-ring. Our objective is to study the structure of certain classes of $D^{*}$-ring.

\section{Main results}

We start by stating the following known lemmas: Lemmas 2.1 and 2.2 were proved in [5], Lemmas 2.3 and 2.4 were proved in [4].

Lemma 2.1. Let $R$ be a weakly periodic ring. Then the Jacobson radical $J$ of $R$ is nil. If, furthermore, $x R \subseteq N$ for all $x \in N$, then $N=J$ and $R$ is periodic.

LEMMA 2.2. If $R$ is a weakly periodic division ring, then $R$ is a field. 
2 Structure of rings with certain conditions on zero divisors

Lemma 2.3. Let $R$ be a periodic ring and $x$ any element of $R$. Then

(a) some power of $x$ is idempotent;

(b) there exists an integer $n>1$ such that $x-x^{n} \in N$.

Lemma 2.4. Let $R$ be a periodic ring and let $\sigma: R \rightarrow S$ be a homomorphism of $R$ onto a ring $S$. Then the nilpotents of $S$ coincide with $\sigma(N)$, where $N$ is the set of nilpotents of $R$.

Definition 2.5. A ring is said to be a $D$-ring if every zero divisor is nilpotent. A ring $R$ is called a $D^{*}$-ring if every zero divisor $x$ in $R$ can be written as $x=a+b$, where $a \in N$, $b \in P$, and $a b=b a$.

Theorem 2.6. A ring $R$ is a $D^{*}$-ring if and only if every zero divisor of $R$ is periodic.

Proof. Assume $R$ is a $D^{*}$-ring and let $x$ be any zero divisor. Then

$$
x=a+b, \quad a \in N, b \in P, a b=b a .
$$

So, $(x-a)=b=b^{n}=(x-a)^{n}$. This implies, since $x$ commutes with $a$, that $(x-a)=$ $(x-a)^{n}=x^{n}+$ sum of pairwise commuting nilpotent elements.

Hence

$$
x-x^{n} \in N \quad \text { for every zero divisor } x
$$

Since each such $x$ is included in a subring of zero divisors, which is periodic by Chacron's theorem, $x$ is periodic.

Suppose, conversely, that each zero divisor is periodic. Then by the proof of $[4$, Lemma $1], R$ is a $D^{*}$-ring.

Theorem 2.7. If $R$ is any normal $D^{*}$-ring, then either $R$ is periodic or $R$ is a $D$-ring. Moreover, $a R \subseteq N$ for each $a \in N$.

Proof. If $R$ is a normal $D^{*}$-ring which is not a $D$-ring, then $R$ has a central idempotent zero divisor $e$. Then $R=e R \oplus A(e)$, where $e R$ and $A(e)$ both consist of zero divisors of $R$, hence (in view of Theorem 2.6) are periodic. Therefore $R$ is periodic.

Now consider $a \in N$ and $x \in R$. Since $a x$ is a zero divisor, hence a periodic element, $(a x)^{j}=e$ is a central idempotent for some $j$. Thus $(a x)^{j+1}=(a x)^{j} a x=a^{2} y$ for some $y \in R$. Repeating this argument, one can show that for each positive integer $k$, there exists $m$ such that $(a x)^{m}=a^{2^{k}} w$ for some $w \in R$. Therefore $a R \subseteq N$.

Corollary 2.8. Let $R$ be a $D^{*}$-ring which is not a $D$-ring. If $N \subseteq C$, then $R$ is commutative. Proof. Since $N \subseteq C, R$ is normal. Therefore commutativity follows from Theorem 2.7 and a theorem of Herstein.

Now, we prove the following result for $D^{*}$-rings.

Theorem 2.9. Let $R$ be a normal $D^{*}$-ring.

(i) If $R$ is weakly periodic, then $N$ is an ideal of $R, R$ is periodic, and $R$ is a subdirect sum of nil rings and/or local rings $R_{i}$. Furthermore, if $N_{i}$ is the set of nilpotents of the local ring $R_{i}$, then $R_{i} / N_{i}$ is a periodic field. 
(ii) If $R$ is Artinian, then $N$ is an ideal and $R / N$ is a finite direct product of division rings.

Proof. (i) Using Theorem 2.7, we have

$$
a R \subseteq N \quad \text { for every } a \in N .
$$

This implies, using Lemma 2.1, that $N=J$ is an ideal of $R$, and $R$ is periodic.

As is well-known, we have

$$
R \cong \text { a subdirect sum of subdirectly irreducible rings } R_{i} \text {. }
$$

Let $\sigma: R \rightarrow R_{i}$ be the natural homomorphism of $R$ onto $R_{i}$. Since $R$ is periodic, $R_{i}$ is periodic and by Lemma 2.4,

$$
N_{i}=\text { the set of nilpotents of } R_{i}=\sigma(N) \text { is an ideal of } R_{i} .
$$

We now distinguish two cases.

Case $11 \notin R_{i}$. Let $x_{i} \in R_{i}$, and let $\sigma: x \rightarrow x_{i}$. Then by Lemma 2.3, $x^{k}$ is a central idempotent of $R$, and hence $x_{i}^{k}$ is a central idempotent in the subdirectly irreducible ring $R_{i}$, for some positive integer $k$. Hence $x_{i}^{k}=0\left(1 \notin R_{i}\right)$. Thus $R_{i}=N_{i}$ is a nil ring.

Case $21 \in R_{i}$. The above argument in Case 1 shows that $x_{i}^{k}$ is a central idempotent in the subdirectly irreducible ring $R_{i}$. Hence $x_{i}^{k}=0$ or $x_{i}^{k}=1$ for all $x_{i} \in R_{i}$. So, $R_{i}$ is a local ring and for every $x_{i}+N_{i} \in R_{i} / N_{i}$,

$$
x_{i}+N_{i}=N_{i} \quad \text { or } \quad\left(x_{i}+N_{i}\right)^{k}=1+N_{i} .
$$

So $R_{i} / N_{i}$ is a periodic division ring, and hence by Lemma $2.2, R_{i} / N_{i}$ is a periodic field.

(ii) Suppose $R$ is Artinian. Using (2.3), $a R$ is a nil right ideal for every $a \in N$. So, $N \subseteq J$. But $J \subseteq N$ since $R$ is Artinian. Therefore $N=J$ is an ideal of $R$ and $R / N=R / J$ is semisimple Artinian. This implies that $R / N$ is isomorphic to a finite direct product $R_{1} \times R_{2} \times \cdots \times R_{n}$, where each $R_{i}$ is a complete $t_{i} \times t_{i}$ matrix ring over a division ring $D_{i}$. Since $R$ is Artinian, the idempotents of $R / J$ lift to idempotents in $R$ [2], and hence the idempotents of $R / J$ are central. If $t_{j}>1$, then $E_{11} \in R_{j}$, and $\left(0, \ldots, 0, E_{11}, 0, \ldots, 0\right)$ is an idempotent element of $R / J$ which is not central in $R / J$. This is a contradiction. So $t_{i}=1$ for every $i$. Therefore each $R_{i}$ is a division ring and $R / N$ is isomorphic to a finite direct product of division rings.

The next result deals with a special kind of $D^{*}$-rings.

Theorem 2.10. Let $R$ be a ring such that every zero divisor $x$ can be written uniquely as $x=a+e$, where $a \in N$ and $e$ is idempotent.

(i) If $R$ is weakly periodic, then $N$ is an ideal of $R$, and $R / N$ is isomorphic to a subdirect sum of fields.

(ii) If $R$ is Artinian, then $N$ is an ideal and $R / N$ is a finite direct product of division rings. 


\section{Structure of rings with certain conditions on zero divisors}

Proof. Let $e^{2}=e \in R, x \in R$, and let $f=e+e x-e x e$. Then $f^{2}=f$ and hence $(e f-e) f=$ 0 . So if $f$ is not a zero divisor, then $e f-e=0$. So $e f=e$, and thus $f=e$, which implies that ex $=$ exe. The net result is ex-exe $=0$ if $f$ is not a zero divisor. Next, suppose $f$ is a zero divisor. Then since

$$
\begin{gathered}
f=(\text { ex }- \text { exe })+e ; \quad \text { ex }- \text { exe } \in N, \text { e idempotent; } \\
f=0+f,
\end{gathered}
$$

it follows from uniqueness that $e x-e x e=0$, and hence $e x=e x e$ in all cases. Similarly $x e=e x e$, and thus

$$
\text { all idempotents of } R \text { are central, and hence } R \text { is a normal } D^{*} \text {-ring. }
$$

(i) Using (2.8), $R$ satisfies all the hypotheses of Theorem 2.9(i), and hence $N$ is an ideal, and $R$ is periodic. Using Lemma 2.2, for each $x \in R$, there exists an integer $k>1$, such that $x-x^{k} \in N$, and hence

$$
(x+N)^{k}=(x+N), \quad k=k(x)>1 .
$$

By a well-known theorem of Jacobson [6], (2.9) implies that $R / N$ is a subdirect sum of fields.

(ii) If $R$ is Artinian, then using (2.8), $R$ satisfies the hypotheses of Theorem 2.9(ii). Therefore $N$ is an ideal and $R / N$ is a finite direct product of division rings.

Theorem 2.11. Let $R$ be a semiprime $D^{*}$-ring with $N$ commutative. Then $R$ is either a domain or a J-ring.

Proof. As in the proof of [3, Theorem 1] we can show that if $a^{k}=0$, then $(a r)^{k}=0$ for all $r \in R$. Therefore, by Levitzki's theorem, $N=\{0\}$. Assume $R$ is not a domain, and let $a$ be any nonzero divisor of zero. Then $a$ is potent and $a R$ consists of zero divisors, hence is a $J$-ring containing $a$. Therefore $[a x, a]=0$ for all $x \in R$, hence $(a x)^{n}=a^{n} x^{n}$ for all $x \in R$, and all $n \geq 2$. For $x$ not a zero divisor, choose $n>1$ such that $a^{n}=a$ and $(a x)^{n}=a x$. Then $a^{n} x^{n}=a x$, so $a\left(x^{n}-x\right)=0$ and $x^{n}-x$ is a zero divisor, hence is periodic. It follows by Chacron's theorem that $R$ is a periodic ring; and since $N=\{0\}, R$ is a $J$-ring.

Example 2.12. Let

$$
R=\left\{\left(\begin{array}{ll}
0 & 0 \\
0 & 0
\end{array}\right),\left(\begin{array}{ll}
1 & 1 \\
1 & 1
\end{array}\right),\left(\begin{array}{ll}
1 & 0 \\
0 & 1
\end{array}\right),\left(\begin{array}{ll}
0 & 1 \\
1 & 0
\end{array}\right)\right\}, \quad 0,1 \in G F(2)
$$

Then $R$ is a normal weakly periodic $D^{*}$-ring with commuting nilpotents. $R$ is not semiprime since the set of nilpotent elements $N$ is a nonzero nilpotent ideal. This example shows that we cannot drop the hypothesis " $R$ is semiprime" in Theorem 2.11.

In Theorem 2.14 below, we study the structure of a special kind of $D^{*}$-rings, the class of rings in which every zero divisor is potent. Recall that a ring is semiperfect [2] if and 
only if $R / J$ is semisimple (Artinian) and idempotents lift modulo $J$. We need the following lemma.

Lemma 2.13. Let $R$ be a ring in which every zero divisor is potent. Then $N=\{0\}$ and $R$ is normal. Moreover, If $R$ is not a domain, then $J=\{0\}$.

Proof. If $a \in N$, then $a$ is a zero divisor and hence potent by hypothesis. So $a^{n}=a$ for some positive integer $n$, and since $a \in N$, there exists a positive integer $k$ such that $0=$ $a^{n^{k}}=a$. So $N=\{0\}$. Let $e$ be any idempotent element of $R$ and $x$ is any element of $R$. Then $e x-$ exe $\in N$, and hence $e x-e x e=0$. Similarly, $x e=e x e$. So $e x=x e$ and $R$ is normal.

Let $x$ be a nonzero divisor of zero. Then $x J$ consists of zero divisors, which are potent. Therefore $x J=\{0\}$. But then $J$ consists of zero divisors, hence potent elements, and therefore $J=\{0\}$.

THEOREM 2.14. Let $R$ be a ring such that every zero divisor is potent.

(i) If $R$ is weakly periodic, then every element of $R$ is potent and $R$ is a subdirect sum of fields.

(ii) If $R$ is prime, then $R$ is a domain.

(iii) If $R$ is Artinian, then $R$ is a finite direct product of division rings.

(iv) If $R$ is semiperfect, then $R / J$ is a finite direct product of division rings.

Proof. (i) Since $R$ is weakly periodic, every element $x \in R$ can be written as

$$
x=a+b, \quad \text { where } a \in N, b \text { is potent. }
$$

But $N=\{0\}$ (Lemma 2.13), so every $x \in R$ is potent and hence $R$ is isomorphic to a subdirect sum of fields by a well-known theorem of Jacobson.

(ii) Suppose $R$ is a prime, then $R$ is a prime ring with $N=\{0\}$, and hence $R$ is a domain.

(iii) Let $R$ be an Artinian ring such that every zero divisor is potent. Since $N=\{0\}$ (Lemma 2.13) and $R$ is Artinian, $J=N=\{0\}$. So $R$ is semisimple Artinian and hence it is isomorphic to a finite direct product $R_{1} \times R_{2} \times \cdots \times R_{n}$, where each $R_{i}$ is a complete $t_{i} \times t_{i}$ matrix ring over a division ring $D_{i}$. If $t_{j}>1$, then $E_{11} \in R_{j}$, and $\left(0, \ldots, 0, E_{11}, 0, \ldots, 0\right)$ is an idempotent element of $R$ which is not central in $R$ contradicting Lemma 2.13. So $t_{i}=1$ for every $i$. Therefore each $R_{i}$ is a division ring and $R$ is isomorphic to a finite direct product of division rings.

(iv) Let $R$ be a semiperfect ring such that every zero divisor is potent. Then $R / J$ is semisimple Artinian and hence it is isomorphic to a finite direct product $R_{1} \times R_{2} \times \cdots \times$ $R_{n}$, where each $R_{i}$ is a complete $t_{i} \times t_{i}$ matrix ring over a division ring $D_{i}$. Since $R$ is semiperfect, the idempotents of $R / J$ lift to idempotents in $R$, and hence the argument of part (iii) above implies that each $R_{i}$ is a division ring and $R / J$ is isomorphic to a finite direct product of division rings.

\section{Acknowledgment}

We wish to express our indebtedness and gratitude to the referee for the helpful suggestions and valuable comments. 


\section{Structure of rings with certain conditions on zero divisors}

\section{References}

[1] H. Abu-Khuzam, H. E. Bell, and A. Yaqub, Structure of rings with a condition on zero divisors, Scientiae Mathematicae Japonicae 54 (2001), no. 2, 219-224.

[2] F. W. Anderson and K. R. Fuller, Rings and Categories of Modules, Graduate Texts in Mathematics, vol. 13, Springer, New York, 1974.

[3] H. E. Bell, Some commutativity results for periodic rings, Acta Mathematica Academiae Scientiarum Hungaricae 28 (1976), no. 3-4, 279-283.

[4] _ A commutativity study for periodic rings, Pacific Journal of Mathematics 70 (1977), no. $1,29-36$.

[5] J. Grosen, H. Tominaga, and A. Yaqub, On weakly periodic rings, periodic rings and commutativity theorems, Mathematical Journal of Okayama University 32 (1990), 77-81.

[6] N. Jacobson, Structure theory for algebraic algebras of bounded degree, Annals of Mathematics 46 (1945), 695-707.

[7] H. Tominaga and A. Yaqub, Some commutativity conditions for left s-unital rings satisfying certain polynomial identities, Results in Mathematics 6 (1983), no. 2, 217-219.

Hazar Abu-Khuzam: Department of Mathematics, American University of Beirut, Beirut 1107 2020, Lebanon

E-mail address: hazar@aub.edu.lb

Adil Yaqub: Department of Mathematics, University of California, Santa Barbara, CA 93106-3080, USA

E-mail address: yaqub@math.ucsb.edu 


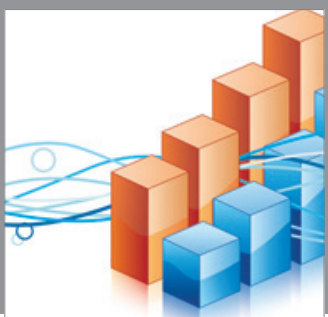

Advances in

Operations Research

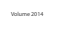

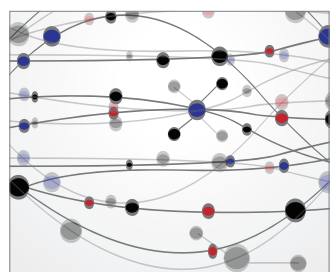

\section{The Scientific} World Journal
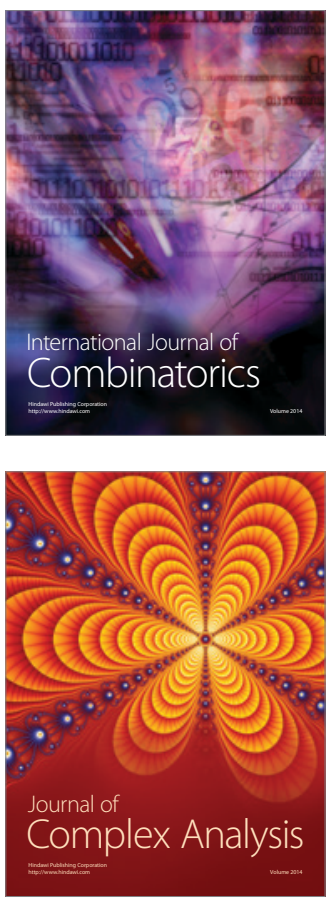

International Journal of

Mathematics and

Mathematical

Sciences
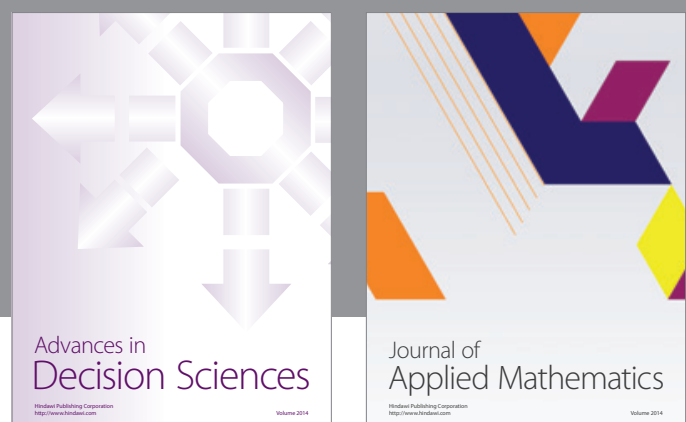

Journal of

Applied Mathematics
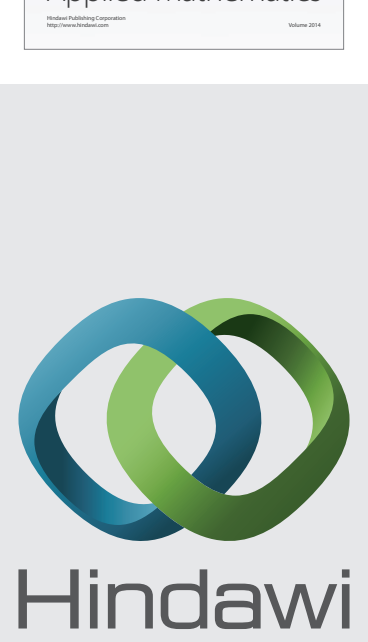

Submit your manuscripts at http://www.hindawi.com
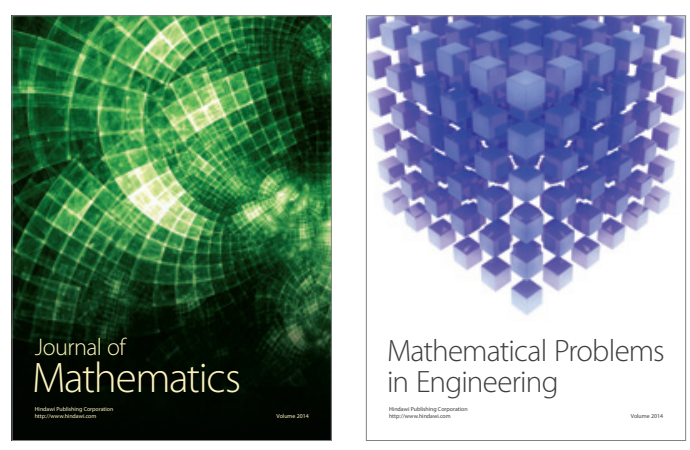

Mathematical Problems in Engineering
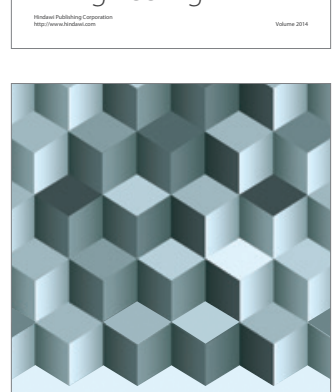

Journal of

Function Spaces
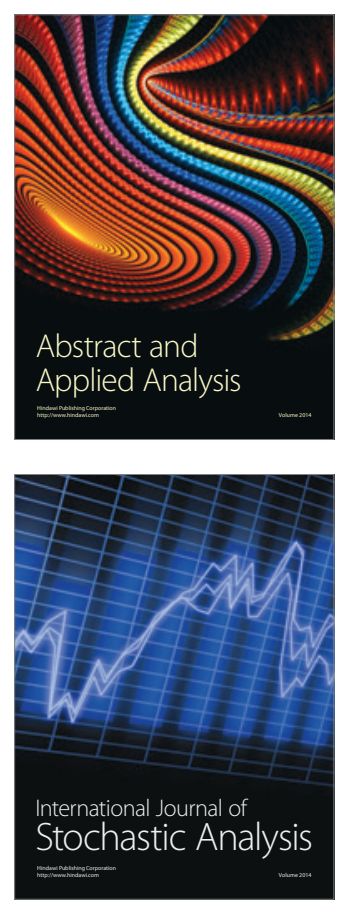

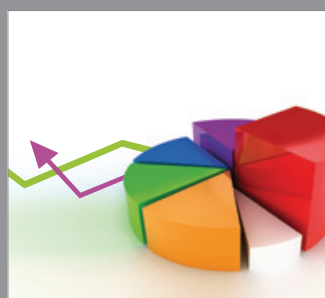

ournal of

Probability and Statistics

Promensencen
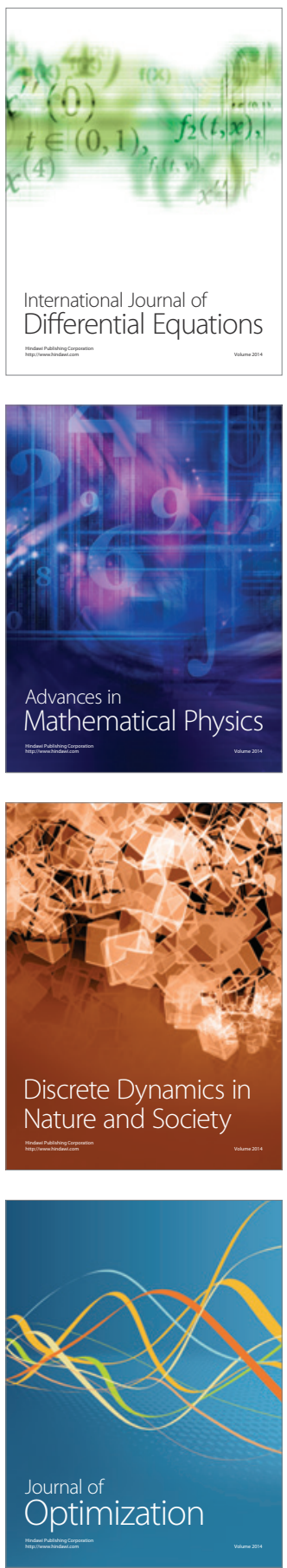